\title{
Phosphorylation of pericyte FAK-Y861 affects tumour cell apoptosis and tumour blood vessel regression
}

\author{
Delphine M. Lees ${ }^{1} \cdot$ Louise E. Reynolds $^{1}\left[\right.$ (1) $\cdot$ Ana Rita Pedrosa ${ }^{1} \cdot$ Marina Roy-Luzarraga ${ }^{1} \cdot$ Kairbaan M. Hodivala-Dilke $^{1}$
}

Received: 21 May 2020 / Accepted: 25 February 2021 / Published online: 17 March 2021

(c) The Author(s) 2021, corrected publication 2021

\begin{abstract}
Focal adhesion kinase (FAK) is a non-receptor tyrosine kinase that is overexpressed in many cancer types and in vivo studies have shown that vascular endothelial cell FAK expression and FAK-phosphorylation at tyrosine (Y) 397, and subsequently FAK-Y861, are important in tumour angiogenesis. Pericytes also play a vital role in regulating tumour blood vessel stabilisation, but the specific involvement of pericyte FAK-Y397 and FAK-Y861 phosphorylation in tumour blood vessels is unknown. Using Pdgfr $\beta C r e+; F A K^{W T / W T}, P d g f r \beta C r e+; F A K^{Y 397 F / Y 397 F}$ and Pdgfr $\beta C r e+; F A K^{Y 861 F / Y 861 F}$ mice, our data demonstrate that Lewis lung carcinoma tumour growth, tumour blood vessel density, blood vessel perfusion and pericyte coverage were affected only in late stage tumours in Pdgfr $\beta C r e+; F A K^{Y 861 F / Y 861 F}$ but not Pdgfr $\beta C r e+; F A K^{Y 397 F / Y 397 F}$ mice. Further examination indicates a dual role for pericyte FAK-Y861 phosphorylation in the regulation of tumour vessel regression and also in the control of pericyte derived signals that influence apoptosis in cancer cells. Overall this study identifies the role of pericyte FAK-Y861 in the regulation of tumour vessel regression and tumour growth control and that non-phosphorylatable FAK-Y861F in pericytes reduces tumour growth and blood vessel density.
\end{abstract}

Keywords Angiogenesis $\cdot$ Cancer $\cdot$ Pericytes $\cdot$ Focal adhesion kinase (FAK)

\section{Introduction}

Angiogenesis is a complex biological process that involves multiple cell types, crosstalk between these cells and responses to different stimuli [1-3]. Interactions between pericytes and endothelial cells play important roles in angiogenesis. Pericyte recruitment to newly forming blood vessels is thought to promote neovessel stabilisation and is an essential step in neovascularisation $[4,5]$. Failure to recruit pericytes to blood vessels can affect vascular remodelling, vessel regression and vessel leakage [1,2,6]. This has led to pericyte-targeted therapies being developed and a better understanding of the role of pericytes in tumour angiogenesis $[7,8]$.

DelphineM. Lees and LouiseE. Reynolds are Joint authorship.

Kairbaan M. Hodivala-Dilke

k.hodivala-dilke@qmul.ac.uk

1 Adhesion and Angiogenesis Laboratory, Centre for Tumour Microenvironment, Barts Cancer Institute - a CR-UK Centre of Excellence, Queen Mary University of London, John Vane Science Centre, Charterhouse Square, London EC1M 6BQ, UK
It is well documented that focal adhesion kinase (FAK) plays a central role in different aspects of tumour growth and is overexpressed in many types of cancer [9-11]. One of the many roles of endothelial cell FAK, in the promotion of tumour growth, is in the initiation of angiogenesis [12]. FAK regulates growth, survival, migration and invasion through its dual role as a kinase and as a scaffolding protein. FAK kinase activity results in tyrosine (Y) 397-phosphorylation, which, in turn, allows proteins containing the Src-homology (SH2) domain to bind to FAK, e.g., Src and PI3K. FAK-Src complexing is thought to potentiate further FAK phosphorylation at other FAK domains including FAK-Y861 [13, 14]. Most studies have pointed towards essential roles for phosphorylation of FAK-Y397 but much less in known about the requirement of FAK-Y861. We and others have shown previously that endothelial cell (EC) FAK is required for tumour growth since EC FAK loss leads to a reduction in tumour growth, accompanied by a reduction in tumour vascular density [12]. Endothelial specific loss of FAK inhibits brain tumour formation and leads to normalisation of the vasculature [15]. Global constitutive deletion of exon 5, which encodes FAK-Y397, also leads to an embryonic lethal phenotype with vascular permeability defects [16]. More 
a

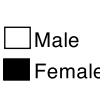

b

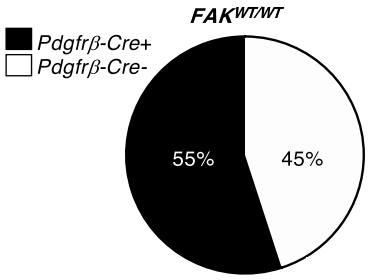

C

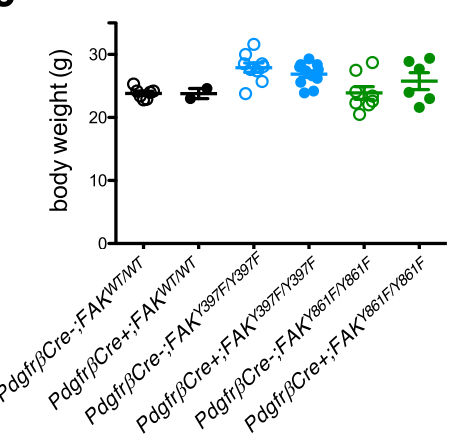

Pdgfr BCre+;FAKWTWT

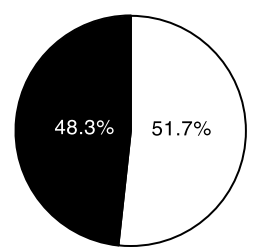

weights 8 wks males e
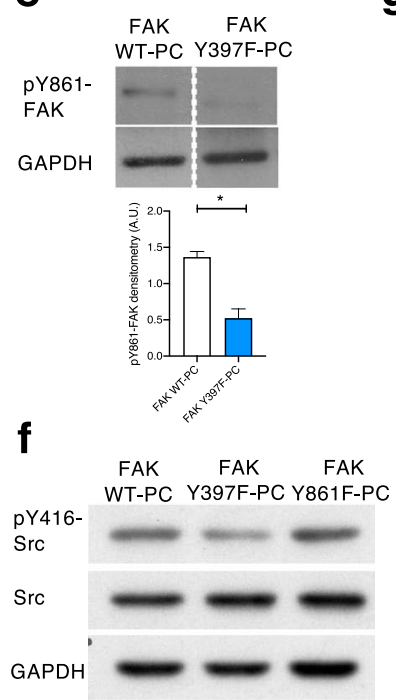

weights 8 wks females

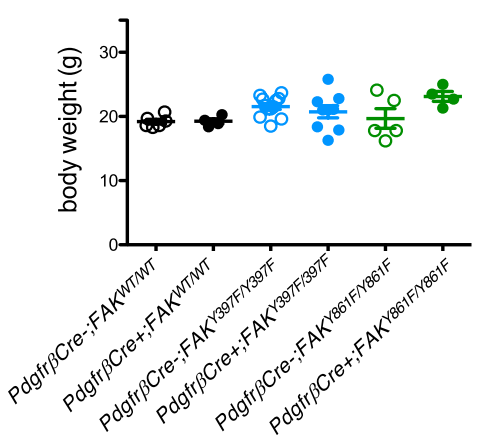

Figure 1. Lees, D.M. et al.
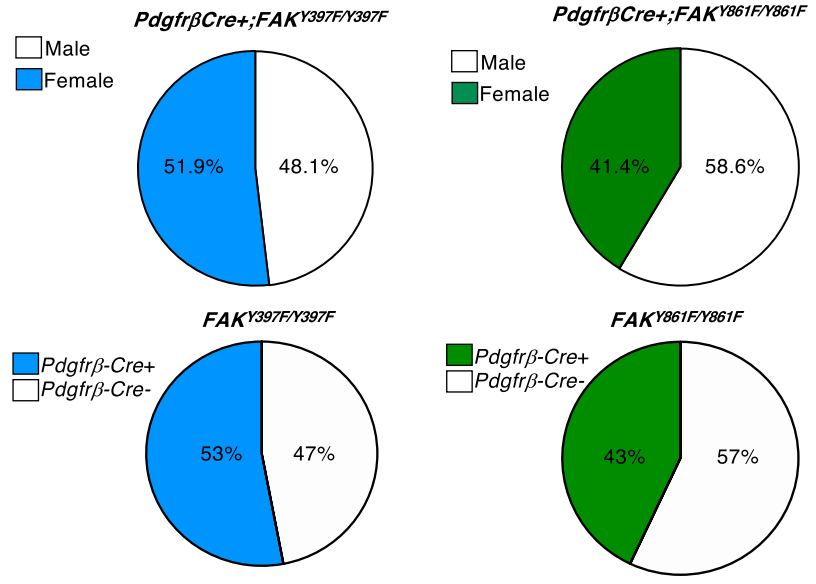

d

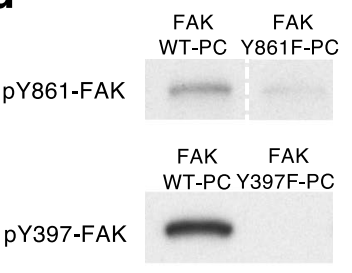

PdgfrßCre+;FAK Y861FI861F

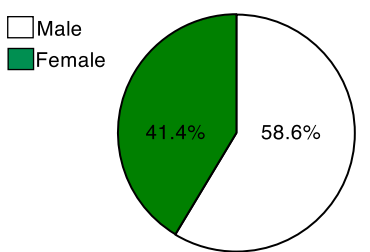

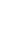


४Fig. 1 LLC tumour growth and angiogenesis are reduced in $\mathrm{Pdgfr} \beta \mathrm{Cre}+; \mathrm{FAK} \mathrm{Y}^{\mathrm{Y} 61 \mathrm{~F} / \mathrm{Y} 861 \mathrm{~F}}$ mice. a Pdgfr $\beta C$ re $+; F A K^{W T / W T}, \quad$ Pdgfr $\beta C r e+; F A K^{Y 397 F / Y 397 F}$ and Pdgfr $\beta C r e+; F A K^{Y 861 F / Y 861 F}$ mice were born at normal male;female ratios; b Mendelian ratios with c similar body weights. Pie chart in a represents percentage male:female ratio, in $\mathbf{b}$ represents \% Cre + and Cre- mice born to each genotype ( $n=60$ mice/ genotype). d Western blotting of primary pericytes isolated from Pdgfr $\beta C r e+; F A K^{W T / W T}, \quad P d g f r \beta C r e+; F A K^{Y 397 F / Y 397 F}$ and Pdgfr $\beta C$ Cre $+; F A K^{Y 861 F / Y 861 F}$ mice confirmed reduced levels of p-Y397 and pY861-FAK in FAKY397F and FAKY861F pericytes, respectively. e pY861-FAK levels are significantly reduced in Y397F pericytes, $n=2$ independent lysates/genotype. Bar chart represents mean pY861-FAK levels + s.e.m. ${ }^{*} P=0.0155$. GAPDH act as loading control. f pY416-Src levels are significantly reduced in Y397FFAK pericytes. Blots shows pY416-Src, total Src and GAPDH loading control. $\mathbf{g}$ In vivo tumour growth was significantly reduced only in Pdgfr $\beta C r e+; F A K^{Y 861 F / Y 861 F}$ mice. Graphs represent mean tumour volume \pm s.e.m.; $n=15$ Pdgfr $\beta C r e+; F A K^{W T W T}$ mice, 14 Pdgfr $\beta C r e+; F A K^{Y 397 F / Y 397 F}$ mice and 11 Pdgfr $\beta C r e+; F A K^{Y 861 F / Y 861 F}$ mice. $* * * * P<0.0001$. Two-sided Mann-Whitney $U$ rank sum test. h Tumour blood vessel density was significantly reduced only in Pdgfr $\beta C r e+; F A K^{Y 861 F / Y 861 F}$ mice. Bar charts represent mean blood vessel density +s.e.m. $* P=0.0498 ;$ ns, not significant; $n=6$ Pdgfr $\beta C r e+; F A K^{W T / W T}$ tumours, 6 Pdgfr $\beta C r e+; F A K^{\text {Y397F/Y397F }}$ tumours and 5 Pdgfr $\beta C r e+; F A K^{Y 861 F / Y 861 F}$ tumours. Two-sided Student's $t$-test. Representative endomucin stained LLC tumour sections are shown for each genotype. Scale bar, $50 \mu \mathrm{m}$

loss of pericyte FAK enhancing tumour angiogenesis [19]. Given that pericytes are an important cell type in the regulation of tumour growth and paracrine signalling [20-22], here we have examined the role of FAK point mutations in pericytes during pathological angiogenesis. Specifically, we have examined the effect of non-phosphorylatable mutations of tyrosines 397 and 861 (Y397F and Y861F) - FAK residues using pericyte-specific FAK ${ }^{\mathrm{Y} 397 \mathrm{~F} / \mathrm{Y} 397 \mathrm{~F}}$ and FAK ${ }^{\mathrm{Y} 861 \mathrm{~F} /}$ Y861F mice.

In this study, we show that in a subcutaneous Lewis Lung Carcinoma (LLC) tumour model, both angiogenesis and tumour growth are reduced only in mice which have the pericyte-specific FAK-Y861F mutation. This correlates with a significant increase in vessel regression. Furthermore, examination of the secretome and protein expression in FAK-Y861F pericytes highlight an altered pericyte signature involving cytokines and protein secretion that are involved in cancer cell apoptosis. Thus, pericyte FAK-Y861 plays a role in the control of tumour growth.

\section{Results}

\section{Generation of pericyte-specific FAK mutant mice}

We generated a new mouse model that enables us to study the effect of endogenous deletion of Y397 and Y861 of FAK in pericytes, during pathological angiogenesis. In this knock-out/knockin mouse model we initially generated myc-tagged chicken-WT-FAK, or non-phosphorylatable -Y397F or -Y861F mutant FAK constructs (preceded by a STOP sequence flanked by loxP sites) targeted to the Rosa26 (R26) locus. These mice were bred with PC-specific Pdgfr $\beta C r e ; F A K^{f l f l}$ mice to generate mutant FAK-knockin and endogenous FAK-knockout in pericytes under Cre control (Supplementary Fig. 1a, b). These mice show no defects in Mendelian ratios, gender distribution or weights and had no obvious adverse phenotype (Fig. 1a-c). Pericytes isolated from Pdgfr $\beta C r e+; F A K^{W T / W T}$, Pdgfr $\beta C r e+; F A K^{Y 861 F / Y 861 F}$ and Pdgfr $\beta C r e+; F A K^{Y 397 F / Y 397 F}$ mice confirmed the presence of the myc-tag indicating chicken FAK-knockin and normal levels of total FAK (Supplementary Fig. 1c). We also confirmed reduced pY397 in FAK-Y397F pericytes and reduced pY861 in FAK-Y861F pericytes (Fig. 1d). Similarly to work published in endothelial cells [17], Y397F mutation in pericytes correlates with reduced levels of pY861F (Fig. 1e). Phosphorylated Src was reduced in Y397F pericytes, but not in Y861F pericytes (Fig. 1f).

\section{Tumour growth and blood vessel density are reduced in Pdgfr $\beta C$ re + ;FAK ${ }^{\text {Y861F/Y861F }}$ but not Pdgfr $\beta C$ Cre + ;FAK ${ }^{\text {Y397F/Y397F mice }}$}

To examine the effects of pericyte FAK-Y397F and Y $861 \mathrm{~F}$ mutations on tumour growth and angiogenesis, $P d g f r \beta C r e+; F A K^{W T / W T}$ control mice, Pdgfr $\beta C r e+; F A K^{\text {Y86IF/Y861F and Pdgfr } \beta C r e+; F A K^{Y 397 F / Y 397 F}}$ mice were injected subcutaneously with Lewis Lung Carcinoma cells (LLC). In vivo tumour growth was reduced in Pdgfr $\beta C r e+; F A K^{Y 861 F / Y 861 F}$, but not Pdgfr $\beta C r e+; F A K^{Y 397 F / Y 397 F}$ mice. Furthermore, the Pdgfr $\beta C r e+; F A K^{\text {Y861F/Y861F but not }}$ Pdgfr $\beta C r e+; F A K^{Y 397 F / Y 397 F}$ mice had significantly reduced blood vessel density (as determined by the number of endomucin-positive vessels per $\mathrm{mm}^{2}$ of age-matched, sizematched tumours) compared with Pdgfr $\beta C r e+; F A K^{W T / W T}$ control mice (Fig. 1g, h).

In addition to the above, blood vessel perfusion (determined by the percentage of endomucin-positive blood vessels that were also positive for PE-PECAM antibody after antemortem perfusion) and pericyte coverage (determined by the percentage of vessels with NG2-positive mural cell association) were both reduced in Pdgfr $\beta C r e+; F A K^{Y 861 F / Y 861 F}$ but not Pdgfr $\beta C r e+; F A K^{Y 397 F / Y 397 F}$ mice (Fig. 2 a, b). Loss of blood vessels can result from partial or persistent regression of vascular endothelial cells. Blood vessel regression is associated with endothelial cell loss and pericyte dropout leaving behind empty collagen IV basement membrane sleeves [23, 24]. Thus, vessel regression can be determined by the presence of endothelial cell-negative, collagen IV-positive basement membrane blood vessel sleeves. Collagen IV / endomucin double-immunostaining 
a
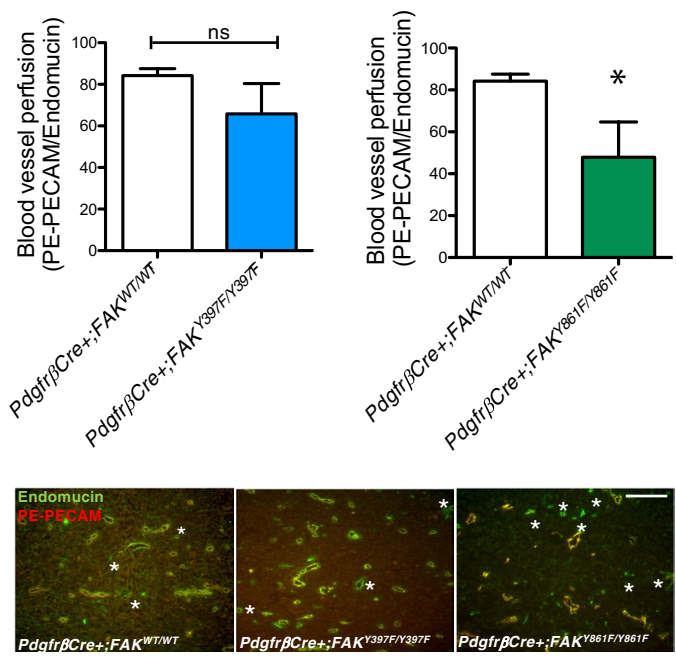

b
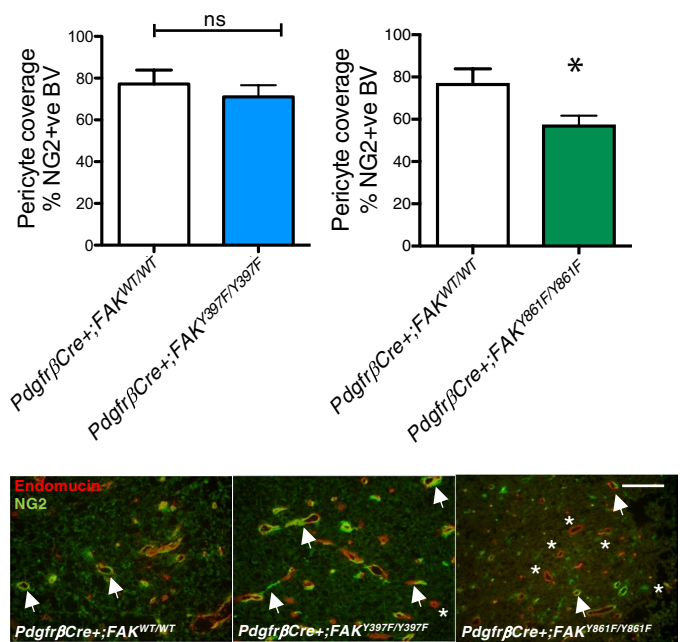

\section{C}
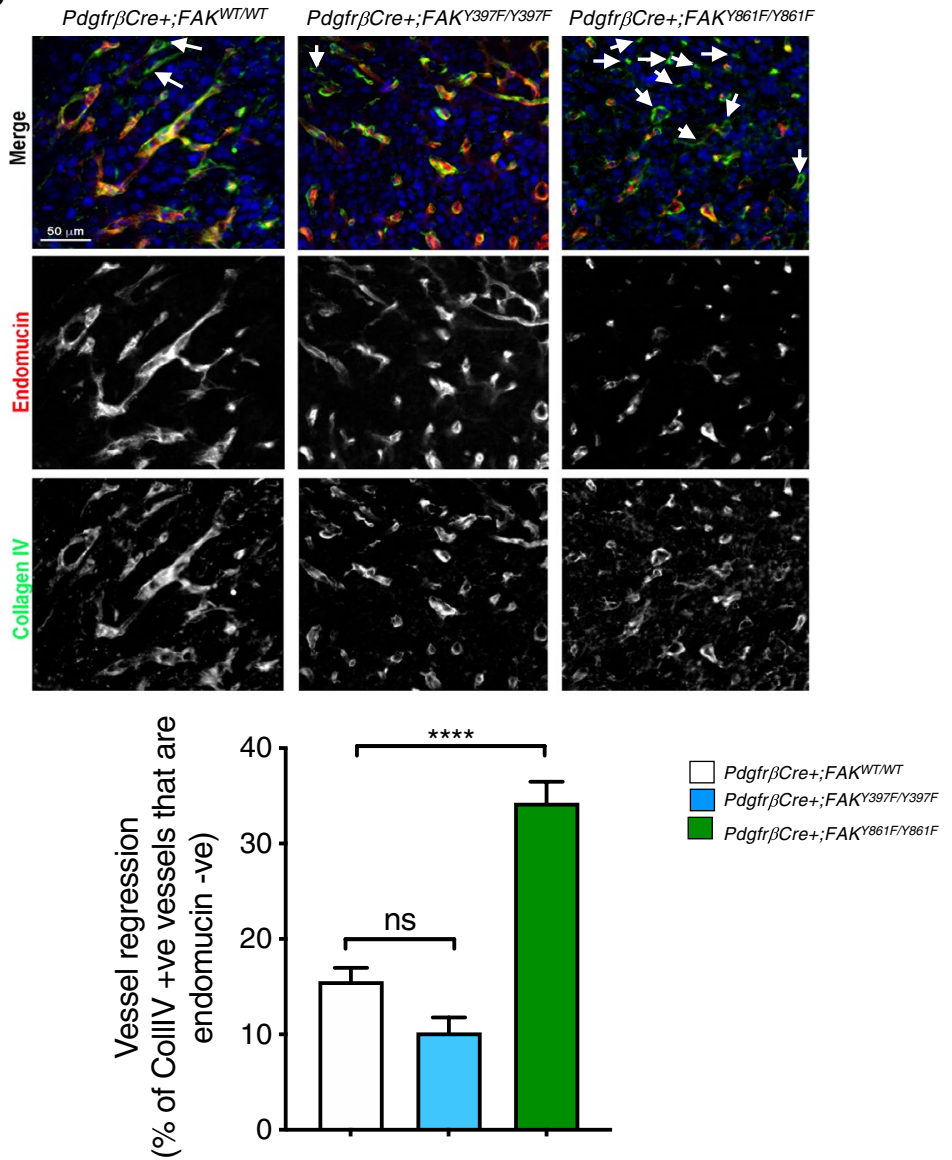

of day 14-21 tumours (when the rate of tumour growth in Pdgfr $\beta C r e+; F A K^{Y 861 F / Y 861 F}$ mice slows down) from Pdgfr $\beta C r e+; F A K^{W T / W T}$, Pdgfr $\beta C r e+; F A K^{Y 397 F / Y 397 F}$ and Pdgfr $\beta C r e+; F A K^{Y 861 F / Y 861 F}$ mice showed a significant increase in endomucin-negative / collagen IV-positive blood vessel sleeves in tumours from $P d g f r \beta C r e+; F A K^{Y 861 F / Y 861 F}$ compared with tumours from Pdgfr $\beta C r e+; F A K^{W T / W T}$, Pdgfr $\beta C r e+; F A K^{Y 397 F / Y 397 F}$ mice (Fig. 2c). These results 
4Fig. 2 Blood vessel perfusion and pericyte coverage are reduced in Pdgfr $\beta C r e+; F A K^{Y 861 F / Y 861 F}$ mice. a PE-PECAM antibody perfused LLC tumours grown in Pdgfr $\beta C r e+; F A K^{W T / W T}$, Pdgfr $\beta C r e+; F A K^{Y 397 F / Y 397 F}$ and Pdgfr $\beta C r e+; F A K^{Y 861 F / Y 86 I F}$ mice showed a significant reduction in functional tumour blood vessels in Pdgfr $B C r e+; F A K^{Y 861 F / Y 861 F}$ mice. Bar charts represent mean blood vessel perfusion + s.e.m. ${ }^{*} P=0.032$; ns, not significant; $n=6$ Pdgfr $\beta C r e+; F A K^{W T / W T}$ tumours, 5 Pdgfr $\beta C r e+; F A K^{Y 397 F / Y 397 F}$ tumours and 4 Pdgfr $\beta C r e+; F A K^{Y 861 F / Y 861 F}$ tumours. Two-sided Student's $t$-test. Representative endomucin stained and PE-PECAM antibody perfused LLC tumour sections are shown for each genotype. asterisks, non-perfused blood vessels. b Pericyte coverage of tumour blood vessels was also reduced in these mice. Bar charts represent mean pericyte coverage + s.e.m. $* P=0.03$, ns, not significant; $n=6$ Pdgfr $\beta C r e+; F A K^{W T / W T}$ tumours, 5 Pdgfr $\beta C r e+; F A K^{Y 397 F / Y 397 F}$ tumours and 4 Pdgfr $\beta C r e+; F A K^{\text {Y861F/Y86IF }}$ tumours. Two-sided Student's $t$-test. Representative double stained endomucin and NG2 LLC tumour sections are shown for each genotype. Arrows, $\mathrm{NG} 2$ + endomucin + blood vessels; asterisks, NG2- endomucin + blood vessels. Scale bar in a and b, 50 $\mu \mathrm{m}$. c Day 14-21 tumours from Pdgfr $\beta C r e+; F A K^{W T / W T}$, Pdgfr $\beta C r e+; F A K^{Y 397 F / Y 397 F}$ and Pdgfr $\beta C r e+; F A K^{Y 861 F / Y 861 F}$ mice were immunostained with Collagen IV and endomucin to identify empty basement membrane sheaths. Bar chart shows vessel regression (\% of Coll IV + vessels that are endomucin -ve) + s.e.m.; $* * * * P<0.0001$; ns, not significant; $n=6$ Pdgfr $\beta C r e+; F A K^{W T / W T}$ mice, 8 Pdgfr $\beta C r e+; F A K^{Y 397 F / Y 397 F}$ mice and 6 Pdgfr $\beta C r e+; F A K^{Y 861 F / Y 861 F}$ mice. Two-way ANOVA. Representative images show Collagen IV and endomucin stained blood vessels from tumours from all genotypes. arrows, Collagen IV + endomucin -ve blood vessels. Scale bar, $50 \mu \mathrm{m}$

correlate with the reduction in tumour growth and suggest that tumour blood vessel regression may be involved in the tumour growth phenotype in Pdgfr $\beta C r e+; F A K^{Y 861 F / Y 861 F}$ mice.

\section{Increased tumour necrosis in early stage tumours of Pdgfr $\beta C r e+; F A K^{Y 861 F / / 861 F}$ mice}

Examination of early stage tumours was undertaken to determine at what stage, during growth of the tumour, angiogenesis was being affected. In early stage tumours (day 12 post tumour cell inoculation) LLC tumour size, blood vessel density and blood vessel perfusion were similar between Pdgfr $\beta C r e+; F A K^{W T / W T}$ and Pdgfr $\beta C r e+; F A K^{Y 861 F / Y 861 F}$ mice (Fig. 3a-c), suggesting that loss of blood vessels occur at a later stage of tumour growth. Indeed to examine whether FAK-Y861F PCs could directly affect the initial stages of microvessel sprouting, aortic rings from Pdgfr $\beta C r e+; F A K^{Y 861 F / Y 861 F}$ and Pdgfr $\beta C r e+; F A K^{W T / W T}$ mice were embedded in collagen and stimulated, or not, with VEGF (30 ng/ml). VEGF treatment significantly increased angiogenic sprouting in both genotypes to the same extent suggesting that mutation of FAK-Y861F in pericytes is not sufficient to directly affect angiogenic sprouting in a tumourfree environment (Supplementary Fig. 2).
Examination of early stage tumours, using $\mathrm{H} \& \mathrm{E}$ stained sections, revealed that tumours from Pdgfr $\beta C r e+; F A K^{Y 861 F / Y 861 F}$ mice had a significant increase in tumour necrosis compared with $P d g f r \beta C r e+; F A K^{W T / W T}$ control mice (Fig. 3d). These results suggest that tumour necrosis precedes the onset of vessel regression and the reduced late stage tumour blood vessel density and tumour growth in Pdgfr $\beta C r e+; F A K^{Y 861 F / Y 861 F}$ mice.

\section{FAK-Y861F pericyte secretome enhances LLC tumour cell apoptosis}

Paracrine or juxtacrine signals from endothelial cells, angiocrine signals, have been implicated in the control of tumour growth where endothelial cell derived signals directly affect tumour cells by altering secreted cytokine profiles $[18,25$, 26]. The role of pericytes in this type of signalling is also emerging $[27,28]$. Thus, we turned our attention to the possible effects of FAK-Y861F pericytes on tumour cell apoptosis. Using R\&D protein profiler arrays, lysates from FAK-Y861F pericytes showed a decrease in levels of thrombospondin, MCP-1, proliferin, TIMP-1 and sICAM/CD54 together with an increase in IGFBP-2, endostatin, TNFalpha, SDF1 and ADAMTS-1 compared with lysates from control WT pericytes. Similar effects on these cytokines were not observed in FAK-Y397F pericytes compared with WT pericytes and SDF-1/CXCL12 was apparently reduced (Fig. 4a). We focused on the role of MCP-1 in the apoptotic phenotype in tumours from Pdgfr $\beta C r e+; F A K^{\text {Y861F/Y861F }}$ mice since, in vitro, MCP-1 promotes mural cell recruitment [29] and in vivo pharmacological inhibition of MCP-1 reduces tumour growth and macrophage recruitment resulting in increased tumour necrosis [30], whilst loss of MCP-1 delays mammary tumourigenesis [31]. Therefore, we treated LLC with MCP-1, after exposure to Y861F conditioned medium (CM), and asked if MCP-1 could be involved in controlling tumour cell apoptosis. CM from FAK-WT and FAKY861F pericytes were incubated with cultured LLC cells and apoptosis quantified by TUNEL staining. Y861F CM caused a significant increase in LLC apoptosis compared with LLCs incubated with WT CM. Indeed the pro-apoptotic phenotype of Y861F CM was reduced by the addition of exogenous recombinant MCP-1 to the pericyte CM (Fig. 4b), suggesting that the reduction of MCP-1 in Y861F pericytes is at least partially involved in the pro-apoptotic phenotype in tumours grown in Pdgfr $\beta C r e+; F A K^{Y 861 F / Y 861 F}$ (Y861F) mice. This pro-apoptotic tumour cell phenotype was confirmed, in vivo, where tumours from Pdgfr $\beta C r e+; F A K^{Y 861 F / Y 861 F}$ mice displayed significantly increased levels of apoptosis compared with tumours from either $P d g f r \beta C r e+; F A K^{W T / W T}$ or Pdgfr $B C r e+; F A K^{Y 397 F / Y 397 F}$ mice (Fig. 4c). 
Fig. 3 Early stage tumour growth is similar between Pdgfr $\beta C r e+; F A K^{W T / W T}$ and Pdgfr $\beta C r e+; F A K^{\text {Y861F/Y861F }}$ mice. Early (day 12): a

Tumour size, b Blood vessel density, and c Blood vessel perfusion were similar between Pdgfr $\beta C r e+; F A K^{W T / W T}$ and Pdgfr $\beta C r e+; F A K^{Y 861 F / Y 861 F}$ mice. Bar charts represent mean + s.e.m.; ns, not significant. Student's $t$-test. For $\mathbf{a}, n=8$ Pdgfr $\beta C r e+; F A K^{W T / W T}$ mice, 5 Pdgfr $B$ Cre + ;FAK ${ }^{Y 861 F / Y 861 F}$ mice. For $\mathbf{b}$ and $\mathbf{c} n=8$ Pdgfr $\beta C r e+; F A K^{W T / W T}$ tumours, 5 Pdgfr $\beta C r e+; F A K^{Y 861 F / Y 861 F}$ tumours. Representative images showing blood vessel perfusion in LLC tumours from $P d g$ fr $\beta C r e+; F A K^{W T / W T}$ and Pdgfr $\beta C r e+; F A K^{Y 861 F / Y 861 F}$ mice. Scale bar, $50 \mu \mathrm{m}$. Asterisks, non-perfused blood vessels. d Tumour necrosis was increased in Pdgfr $\beta C r e+; F A K$ Y861F/Y861F. Bar chart shows

$\%$ necrotic area in tumours from Pdgfr $\beta C r e+; F A K^{W T / W T}$ and Pdgfr $\beta C r e+; F A K$ Y861F/Y861F mice. $* P<0.05$

Wilcoxon test; $n=6$ tumours from Pdgfr $\beta C r e+; F A K^{W T / W T}$ mice and 7 tumours from Pdgfr $\beta C r e+; F A K^{Y 861 F / Y 861 F}$ mice
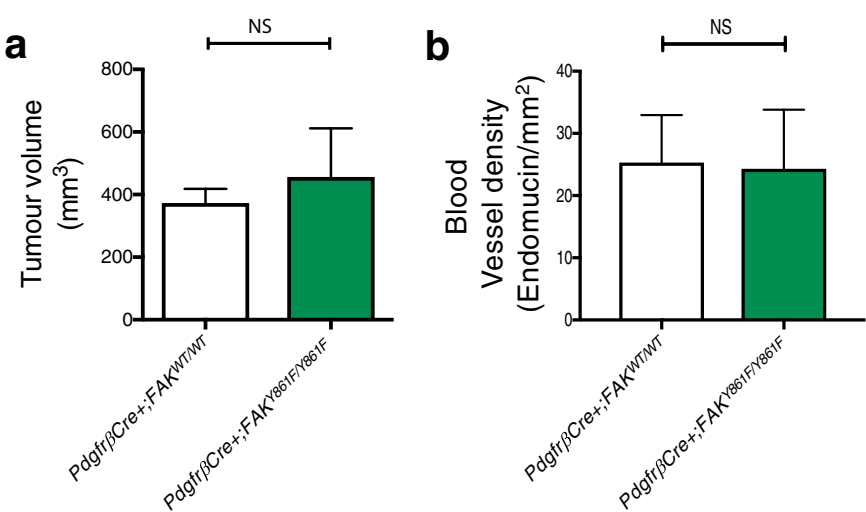

\section{C}
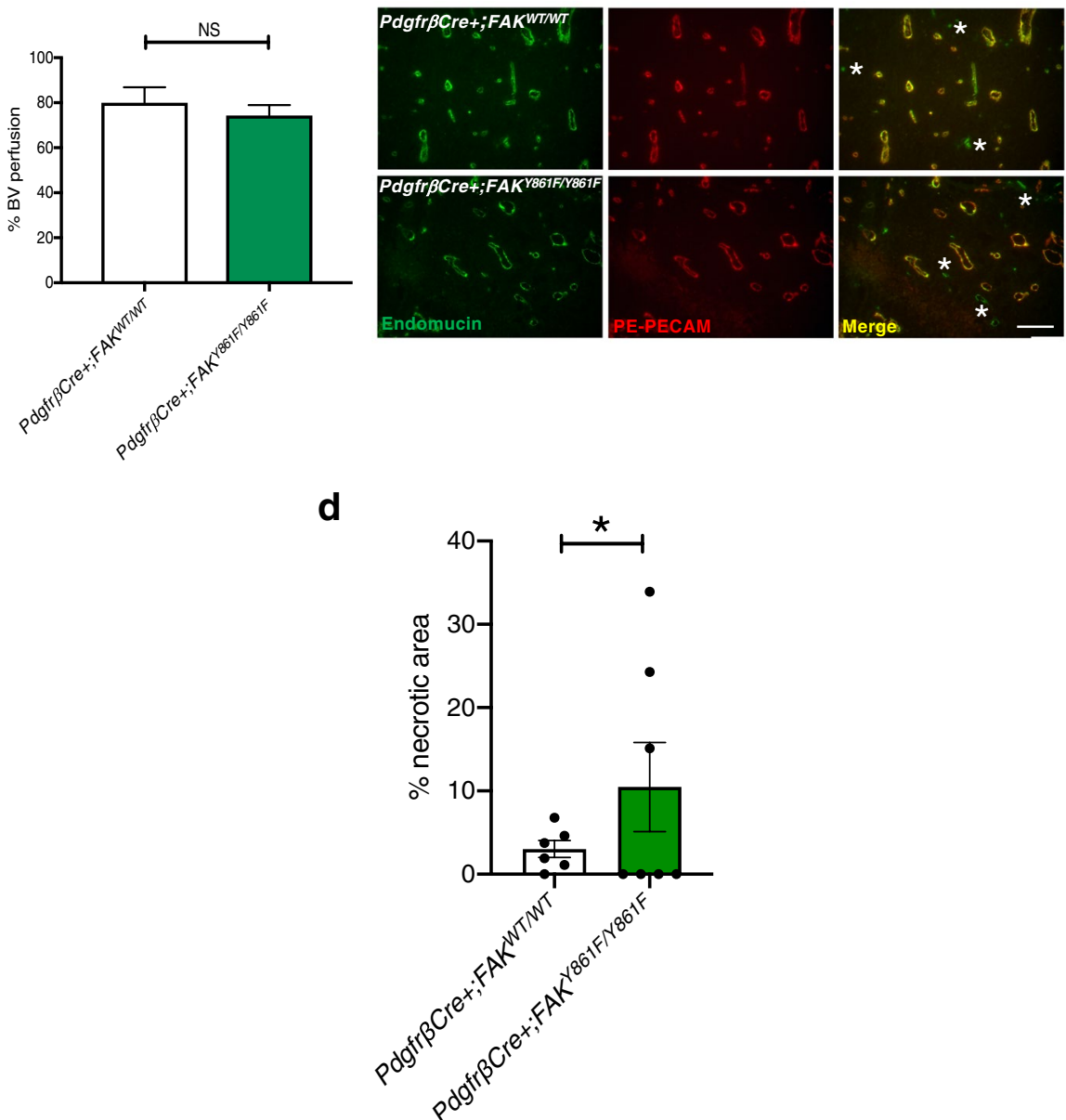

FAK kinase activity leads to phosphorylation of Y397FAK which, in turn, allows protein containing the Srchomology (SH2) domain to bind to FAK, including Src. FAK-Src complexing are thought to potentiate further FAK phosphorylation at other FAK domains including Y861. Since phosphorylated Src was not reduced in Y861F pericytes, we wanted to determine if the phenotype observed in Y861F pericytes was dependent on Src. To address this, Src levels were depleted in FAK-WT, FAKY397F and FAK-Y861F pericytes followed by examination of the effect of CM from these cells on LLC cell death.
CM from Src-depleted FAK-WT and FAK-Y397F pericytes significantly increased LLC apoptosis. Conversely, CM from FAK-Y861 Src-depleted pericytes (Supplementary Fig. 3) had no additional effect on LLC apoptosis above LLC exposed to non-treated CM (Fig. 4d).

These results imply a direct regulation of tumour cells through pericyte paracrine signalling - a direct effect on vascular disruption and ultimately tumour growth that is regulated by FAK-Y861 phosphorylation, and independently of Src. 


\section{Discussion}

Our data demonstrate that FAK-Y861F pericytes decrease tumour burden, at least in part, by directly affecting tumour cell apoptosis, and call for further consideration of the role of tumour pericytes in the direct control of tumour growth, in addition to their effects on vessel stabilisation. Indeed, we have recently shown that pericytes play a central role in the control of primary tumour growth, through cross-talk with multiple cell types in the tumour microenvironment $[19,20]$.

FAK is upregulated in many cancer types and is currently being targeted as a potential anti-cancer agent because of its essential roles in tumour growth and angiogenesis [12, 32-34]. Our previous work identified distinct roles for FAK-Y397 and FAK-Y861 phosphorylation in tumour endothelial cells where endothelial cell FAK-Y397 but not FAK-Y861 reduced tumour growth and angiogenesis, and linking these tyrosine domains to specific signaling pathways downstream of FAK and regulation of an inside out signal affecting endothelial cells surface receptor expression $[17,35]$. Pericytes are thought to be essential in stabilising the tumour vasculature and thus attention has been given to the potential of targeting pericytes to induce vascular disruption in cancer control [36]. However, recent conflicting studies have emerged challenging this idea [37] and suggest possible additional roles for pericytes in regulating tumour growth. In contrast to the effect of FAK-Y397F mutation in endothelial cells [17], our mouse models show that phosphorylation of FAKY397 in pericytes is not a prerequisite for the control of LLC tumour growth or angiogenesis. The reason for this apparent discrepancy likely reflect a different requirement for FAK-Y397-phosphorylation in these two cells types in tumour blood vessels. Additionally, complete loss of pericyte FAK is compensated by an increase in pericyte p-Pyk2, which is sufficient to drive a downstream signaling cascade to promote tumour growth, through effects on both malignant cells and angiogenesis [19]. Importantly, one cannot directly compare pericyte FAK-KO with pericyte FAK- Y397F, since phosphorylation of Src is not affected in pericyte FAK-KO mice, whereas in FAK-Y397F mice, the kinase domain is not affected (as it acts upstream of Y397 in the signaling cascade of FAK activation).

FAK-Y861F mutation in pericytes is associated with reduced tumour growth and angiogenesis that is preceded by an increased tendency for tumour cell necrosis and subsequent blood vessel regression. Since tumour necrosis has been demonstrated to have vascular disrupting effects $[38,39]$ it is tempting to speculate that the early stage enhanced tumour necrosis may initiate vascular disruption. Additionally, given that the Y861F mutation reduces pericyte association with tumour blood vessels, it is likely that vessel stability and maturation is compromised and this reduced pericyte coverage is also likely to be responsible for the decrease in the numbers of functional blood vessels and reduced tumour growth.

Studies have shown that vascular endothelial cells can control tumour growth via angiocrine signals, including cytokines and chemokines $[18,40]$. In our study, the protein signature of FAK-Y861F pericytes is a combination of reduced levels of TSP-2, MCP-1, proliferin, TIMP-1 and sICAM/CD54 together with an increase in IGFBP-2, endostatin, TNF-alpha, SDF1 and ADAMTS-1. This molecular signature associates with pro-apoptotic effects on tumour cells but apparently has little effect on VEGF-stimulated angiogenic sprouting in aortic ring assays in a tumour-free context. The lack of an effect on VEGF-induced aortic ring sprouting suggests that the effect of FAK-Y861F pericyte enhancement on tumour cell apoptosis may be a contributing factor to subsequent vessel regression in a tumour environment. The anti-angiogenic effect of TSP-2 is blocked by VEGF thus providing a possible explanation of why VEGF-stimulated angiogenesis is not affected in aortic ring assays from $P d g f r \beta C r e+; F A K^{Y 861 F / Y 861 F}$ mice where the decrease in TSP-2 might otherwise have increased angiogenesis [41]. The pro-tumoural effects of MCP-1, proliferin, TIMP-1 and soluble ICAM-1 [42-45] whilst the anti-cancer effects of IGFBP-2, endostatin and ADAMTS-1 [46-48] have been published and suggest that this secretome signature may well provide a combination of effects on cancer growth. Indeed MCP-1 addition to conditioned medium from FAK-Y861F pericytes was able to rescue the pro-apoptotic effect of this cocktail on tumour cells.

Furthermore, we have shown here that loss of pericyte FAKY861 phosphorylation intrinsically regulates a paracrine signal that controls apoptosis of tumour cells, independent of Src and does not affect phosphorylation of Y416-Src. We and others have previously shown that loss of FAK, or a non-phosphorylatable Y397F mutation in endothelial cells, disrupts active SrcFAK interactions, driving Src degradation, possibly activating other FAK-Src independent signalling pathways [17, 49-51].

The molecular mechanism by which FAK-Y861 affects this secretome signature is the subject of the future study. Our data support the notion that pericytes are not simply vessel supporting cells, but that via FAK-Y861, can regulate tumour growth via pericyte derived signals directly affecting tumour cell apoptosis.

\section{Methods}

\section{Mice}

To examine the effect of pericyte-specific FAK mutations in vivo, we developed Pdgfr $\beta C r e ; F A K^{W / W T}$ mice 

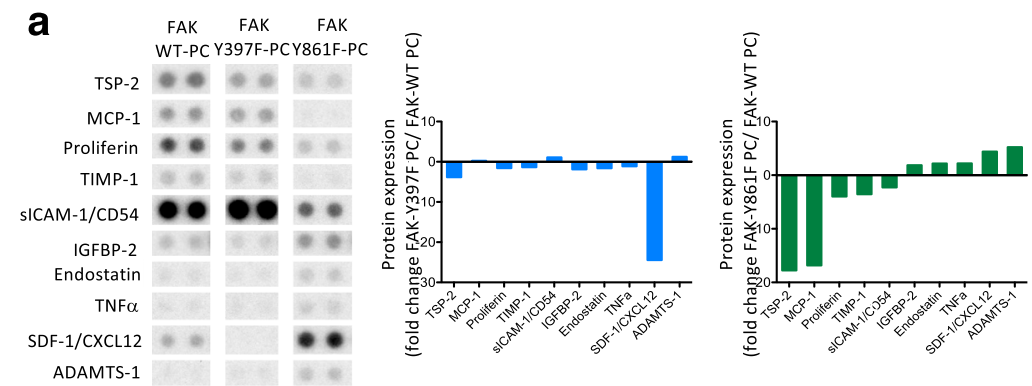

b
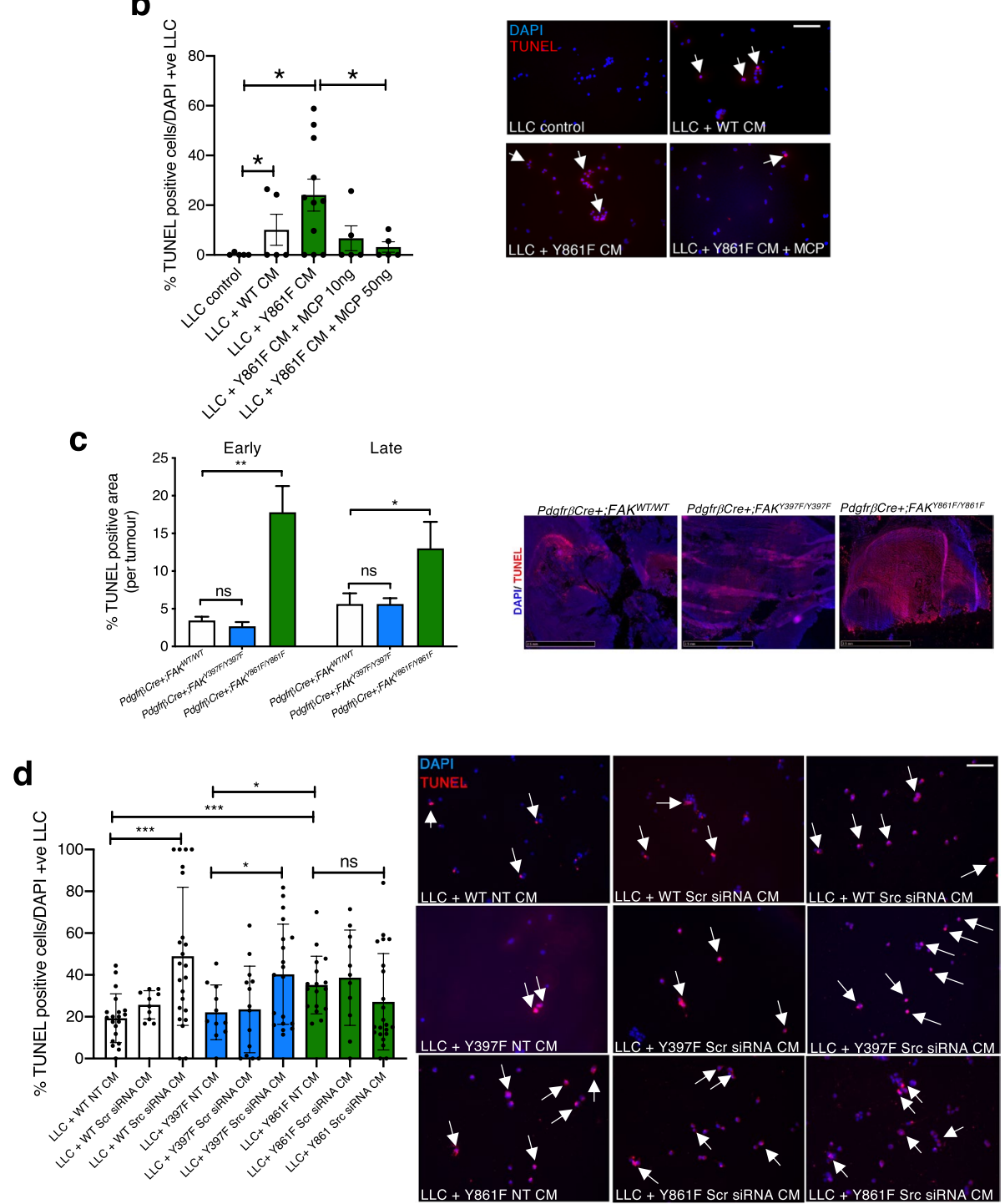

(FAK-WT), Pdgfr $\beta C r e ; F A K^{Y 397 F / Y 397 F}$ (Y397F) mice and Pdgfr $\beta C r e ; F A K^{Y 861 F / Y 861 F}$ (Y861F) mice [52]. All experiments were approved by United Kingdom Home Office Regulations. For animals bred in-house, health screens (quarterly) were conducted in accordance with FELASA guidelines for health monitoring of rodent colonies, to confirm their free status of known pathogens in accordance with FELASA screens. No clinical signs were detected. Animals were housed in groups of 4-6 mice per individually ventilated cage in a $12 \mathrm{~h}$ light dark cycle (06:30-18:30 light; 18:30-06:30 dark), with controlled room temperature $\left(21 \pm 1^{\circ} \mathrm{C}\right)$ and relative humidity $(40-60 \%)$. The cages contained $1-1.5 \mathrm{~cm}$ layer of animal bedding, and with environmental enrichment including cardboard Box-tunnel and 
4Fig. 4 FAK-Y861F pericytes induce apoptosis in LLC tumour cells. a R\&D proteome profiler array using lysates from FAK-WT, FAK-Y397F and FAK-Y861F pericytes. Representative dots of differentially expressed proteins are given. Bar charts show mean fold change in protein expression relative to levels in WT. $N=2$ dots from 1 experiment. b Lewis lung carcinoma (LLC) cells incubated with conditioned medium $(\mathrm{CM})$ from pericytes plus or minus recombinant MCP-1. Treatment with FAK-Y861F pericyte CM increased LLC apoptosis, compared with CM from FAK-WT pericytes. This effect was rescued upon treatment with MCP-1 (10 and $50 \mathrm{ng} / \mathrm{ml}$ ). Bar chart represents \% TUNEL-positive cells + s.e.m. Representative images show effect of $\mathrm{CM}$ and MCP-1 on LLC cells. Arrows, TUNEL-positive cells. $* P<0.05$. One-way ANOVA. $N=5-11$ fields of view/genotype. Scale bar, $500 \mu \mathrm{m}$. c Early and late stage tumours from Pdgfr $\beta C r e+; F A K^{\text {Y861F/Y86IF mice had }}$ significantly larger TUNEL-positive areas than tumours from Pdgfr $\beta C r e+; F A K^{W T / W T}$ and Pdgfr $\beta C r e+; F A K^{Y 397 F / Y 397 F}$ mice. Bar chart shows $\%$ TUNEL-positive area/tumour + s.e.m. $* * P=0.0012$, * $P=0.0464 ;$ ns, not significant; $n=6$ Pdgfr $\beta C r e+; F A K^{W T / W T}$ mice, 8 Pdgfr $\beta C r e+; F A K^{Y 397 F / Y 397 F}$ mice and 6 Pdgfr $\beta C r e+; F A K^{Y 861 F / Y 861 F}$ mice. Two-way ANOVA. Representative images show TUNEL-positive staining in tumours. Scale bar, $2.5 \mathrm{~mm}$. d LLC cells incubated with CM from pericytes treated with Src siRNA, non-specific scrambled siRNA (Scr) or transfection reagent (NT). CM from non-treated (NT) Y861F had a significantly higher effect on LLC apoptosis compared with CM from either WT or Y397F pericytes. Knockdown of Src in both WT and Y397F pericytes significantly increased LLC apoptosis. Src knockdown in Y861F pericytes did not increase LLC apoptosis above control levels. Bar chart represents \% TUNEL-positive cells \pm s.e.m. $* * * P=0.0006, * P=0.0196$ One -way ANOVA. $* * * P=0.0006$ WT NT vs. $861 \mathrm{~F} \mathrm{NT}, * P=0.03$ (Y397F NT vs. Y861F NT). Two-sided students $t$ test. $N=10-23$ fields of view. Scale bar, $500 \mu \mathrm{m}$; arrows in $\mathbf{b}$ and $\mathbf{d}$ indicate TUNEL-positive cells

crinkled paper nesting material. Animals had access to food and water ad libitum.

\section{Tumour growth}

Male and female mice aged 12-16 weeks were injected subcutaneously with $0.5 \times 10^{6}$ Lewis lung carcinoma cells (LLC, ATCC) into the flanks. Tumour dimensions were measured over time and tumour growth was determined using the formula: length $\mathrm{x}$ width ${ }^{2} \times 0.52$. When tumours reached the maximum legal size allowed, mice were killed, tumour sizes measured and tumour samples were either snap-frozen, fixed in $4 \%$ paraformaldehyde (PFA) or fixed in 4\% PFA/sucrose for histological analysis. For early tumour growth studies, tumours were harvested at day 12 post tumour cell injection.

\section{Blood vessel density}

Five $\mu \mathrm{m}$ frozen tumour sections were air-dried for $10 \mathrm{~min}$, washed once in PBS, fixed in acetone for $10 \mathrm{~min}$ at $-20{ }^{\circ} \mathrm{C}$, washed in PBS three times and then blocked with 5\% normal goat serum for $30 \mathrm{~min}$ at room temperature. After blocking, sections were incubated with primary antibodies overnight at $4{ }^{\circ} \mathrm{C}$. Primary antibodies used were directed against endomucin (clone V.7C7; Santa Cruz; sc-65495, 1:100).
Sections were then washed with PBS and incubated with Alexa-Fluor@-conjugated secondary antibody (1:100, Invitrogen) for $45 \mathrm{~min}$ at room temperature before mounting the slides with Prolong® Gold anti-fade reagent (Invitrogen, P36934). Tumour blood vessels were counted across entire midline sections, and the numbers were expressed as vessels/ $\mathrm{mm}^{2}$. For examination of blood vessel regression, tumours were fixed with $4 \% \mathrm{PFA} /$ sucrose then frozen in OCT.

\section{Blood vessel perfusion}

For analysis of the $\%$ of functional tumour vessels, $100 \mu \mathrm{l}$ PE-PECAM antibody (clone 390, neat; Biolegend, 102,407) was injected via the tail vein 10 min prior to culling mice. Tumours were dissected immediately, snap-frozen and sectioned. Frozen sections were then immunostained for endomucin, as described above. To calculate the $\%$ number of functional vessels, the number of PE-PECAM-positive blood vessels was divided by the total number of endomucin-positive blood vessels.

\section{Pericyte coverage}

Frozen tumour sections were double immunostained as described in "Blood vessel density" using the pericytespecific antibody NG2 (AB5320; Millipore, 1:100) and endomucin. The percentage of endomucin-positive vessels with NG2-positive cells associated was calculated.

\section{Blood vessel regression/Collagen IV staining}

Frozen tumour sections were air-dried for $30 \mathrm{~min}$ at room temperature, permeabilised for 3 min with $\mathrm{PBS}+0.5 \%$ TritonX-10 then blocked with 5\% BSA/PBS for $1 \mathrm{~h}$ at room temperature. Sections were then incubated overnight at $4{ }^{\circ} \mathrm{C}$ with Collagen IV (Abcam; 1:100 dilution, ab6586) and endomucin (clone V.7C7, Santa Cruz; 1:100) antibodies. The following day, sections were washed three times with PBS and incubated for $1 \mathrm{~h}$ at room temperature with AlexaFluor conjugated secondary antibodies (1:100). Finally, sections were washed twice in PBS and once in distilled water then mounted (Prolong Gold with DAPI) with a glass slide and images were acquired using a confocal spinning disk microscope and sCMOS confocal camera (Nikon). Image analysis was performed using ImageJ software by making maximum intensity projections of $\mathrm{z}$ stacks and the numbers of vessels counted manually.

\section{Tumour necrosis}

Early stage LLC tumours were fixed in $4 \%$ formaldehyde and bisected. Tumour sections were H\&E stained, scanned using a Panoramic scanner and the area of necrosis (as identified 
by acellular regions of tumour tissue) quantified with ImageJ software.

\section{TUNEL staining}

Tumour cell apoptosis was examined in frozen tumour sections using the In Situ Cell Death Detection Kit, TMR red (Roche, 12156792910) following the manufacturer's instructions. Stained sections were scanned using a Panoramic scanner and the TUNEL-positive area was calculated as the percentage of TUNEL-positive area/ tumour area.

\section{LLC and pericyte conditioned medium experiments}

LLC were plated on coverslips at a density $5 \times 10^{4}$ cells in DMEM $+10 \%$ FCS. At the same time WT and $861 \mathrm{~F}$ pericytes were grown to 50-60\% confluency and conditioned medium (CM) removed. CM was centrifuged to remove cell debris and added to LLCs after removal of DMEM and two PBS washes. Recombinant mouse MCP-1 (Biotechne, MAB479) was added to the CM at either 10 or $50 \mathrm{ng} / \mathrm{ml}$. Cells were incubated with CM for 24 or $48 \mathrm{~h}$ after which cells were stained to detect DNA fragmentation in apoptosis using the BrdU-Red DNA (TUNEL) kit (Abcam, ab66110) following the manufacturer's instructions. Within $3 \mathrm{~h}$ of staining the cells were analysed for BrdU using a Zeiss microscope and Axiovision software. The percentage of TUNEL-positive cells was calculated by counting the total number of cell nuclei and the number of nuclei that were TUNEL positive. For Src siRNA experiments, WT, Y397F and Y861F pericytes were transfected for $48 \mathrm{hr}$ with SMARTpool Src siRNA or non-targeted siRNA (both 10uM final concentration) as a control. After $48 \mathrm{hr}$ cells were lysed for protein extraction to measure Src levels by Western blot analysis. Conditioned medium was also removed and added to LLCs as described above, prior to TUNEL staining.

\section{Primary cell cultures}

Primary mouse brain pericytes were isolated from the mice, cultured and characterised as previously described $[53,54]$. Briefly, brains were removed from mice, minced, digested for $1 \mathrm{~h}$ in $0.1 \%$ collagenase, centrifuged in the presence of $22 \%$ BSA, and cultured in endothelial cell growth media (pMLEC) with the medium changed every 3 days. On reaching confluency, cultures were harvested with trypsin and passaged. During the first two passages, pericyte cultures were grown in pMLEC, and on the third passage they were grown in pericyte medium (PM; ScienCell Research Laboratories) containing 2\% FBS and antibiotics. Tissue culture plates for all experiments were coated with a mixture of collagen $(30 \mu \mathrm{g} / \mathrm{ml})$, gelatin $(0.1 \%)$ and fibronectin $(10 \mu \mathrm{g} / \mathrm{ml})$.

\section{Aortic ring assay}

Aortic rings were isolated from all mouse genotypes as previously described [55].

\section{Angiogenesis and cytokine arrays}

Pericyte angiogenesis and cytokine profiles were compared using the angiogenesis array (ARY015, R\&D Biosystems) and cytokine array (ARY006, R\&D Biosystems). Briefly, cell lysates were prepared as follows: sample buffer was added to the cell culture, the cells were scraped and transferred into a $1.5 \mathrm{ml}$ Eppendorf tube. After sonication, samples were adjusted to the array conditions and mixed with a Detection Antibody Cocktail as indicated by the manufacturer's instructions. Lysates were incubated overnight at $4{ }^{\circ} \mathrm{C}$ on dot-blot membranes. Membranes were washed, incubated with streptavidin-HRP for $30 \mathrm{~min}$ at RT, washed again and ECL was applied to the membrane to reveal the dots. Quantification of dot intensity was performed using Image ${ }^{\mathrm{TM}}$ software.

\section{Western blot analysis}

Primary brain pericytes were grown to $70-80 \%$ confluency then lysed in RIPA buffer. 15-30 $\mu \mathrm{g}$ protein was run on $8 \%$ polyacrylamide gels then transferred to nitrocellulose membranes. Membranes were probed with primary antibody overnight at $4{ }^{\circ} \mathrm{C}$. Myc-tag (Cell Signaling, clone 9B11, 2276, 1:1000), total FAK (Cell Signaling, 3258, 1:1000), phospho-397 FAK (Invitrogen, 44-624G, 1:1000), phospho-861 FAK (Invitrogen, 44-626G, 1:1000), PDGFR $\beta$ (Cell Signaling, clone 28E10, 3169, 1:1000), endomucin (V7.C7, Santa Cruz, 1:1000), Src (Cell Signaling, 2108, 1:1000), pSrc (Cell Signaling, 2101, 1:1000). The anti-HSC70 (Santa Cruz, clone B-6, sc-7298) or GAPDH (Millipore, MAB374) antibody, for loading controls, were used at 1:5000 dilution. Densitometric readings of band intensities were obtained using the Image $J^{\mathrm{TM}}$ software.

\section{Statistical analysis}

Statistical significance was calculated using Prism 8 software and $P<0.05$ was considered statistically significant, unless otherwise indicated. For tumour growth statistics, non-parametric two-sided Mann-Whitney $U$ rank sum test was performed to compare tumour volumes each day. Oneway ANOVA was performed for the in vitro TUNEL and aortic ring assay, two-way ANOVA for the blood vessel 
regression and in vivo TUNEL study. Wilcoxon test was performed for tumour necrosis.

Supplementary Information The online version contains supplementary material available at https://doi.org/10.1007/s10456-021-09776-8.

Acknowledgements We would like to thank Julie Holdsworth and Bruce Williams from Barts Cancer Institute (QMUL, UK) for their expert technical assistance and Professor Ralf Adams (Max Planck Institute for Molecular Biomedicine, Münster, Germany) for the kind gift of the Pdgfr $\beta C r e$ mice.

Author contributions DL designed and executed the experiments. LER wrote the paper, executed the experiments, analysed data and gave scientific advice. RP performed collagen IV staining, in vivo TUNEL staining and analysis and contributed to writing the paper. MR-L analysed tumour necrosis slides. KMH-D conceived and supervised the project and wrote the paper.

Funding This work was supported by a Cancer Research UK Grant (C82181/A12007) and a Radiation Research Unit at the Cancer Research UK City of London Centre Award (C7893/A28990).

Data availability The data that support the findings of this study are available from the corresponding author upon request.

\section{Compliance with ethical standards}

Conflict of interest KHD is a scientific advisor for Ellipses. KHD is joint applicant on Patent claims N421127GB and N417173GB. The other authors declare no conflicts of interest.

Ethical approval All procedures were approved by our local animal ethics committee, Queen Mary University of London, and were executed in accordance with United Kingdom Home Office regulations.

Open Access This article is licensed under a Creative Commons Attribution 4.0 International License, which permits use, sharing, adaptation, distribution and reproduction in any medium or format, as long as you give appropriate credit to the original author(s) and the source, provide a link to the Creative Commons licence, and indicate if changes were made. The images or other third party material in this article are included in the article's Creative Commons licence, unless indicated otherwise in a credit line to the material. If material is not included in the article's Creative Commons licence and your intended use is not permitted by statutory regulation or exceeds the permitted use, you will need to obtain permission directly from the copyright holder. To view a copy of this licence, visit http://creativecommons.org/licenses/by/4.0/.

\section{References}

1. Armulik A, Genove G, Betsholtz C (2011) Pericytes: developmental, physiological, and pathological perspectives, problems, and promises. Dev Cell 21(2):193-215

2. Bergers G, Song S (2005) The role of pericytes in blood-vessel formation and maintenance. Neuro Oncol 7(4):452-464

3. Geevarghese A, Herman IM (2014) Pericyte-endothelial crosstalk: implications and opportunities for advanced cellular therapies. Transl Res 163(4):296-306

4. von Tell D, Armulik A, Betsholtz C (2006) Pericytes and vascular stability. Exp Cell Res 312(5):623-629
5. Stratman AN, Davis GE (2012) Endothelial cell-pericyte interactions stimulate basement membrane matrix assembly: influence on vascular tube remodeling, maturation, and stabilization. Microsc Microanal 18(1):68-80

6. Hellstrom M, Gerhardt H, Kalen M et al (2001) Lack of pericytes leads to endothelial hyperplasia and abnormal vascular morphogenesis. J Cell Biol 153(3):543-553

7. Chen M, Lei X, Shi C et al (2017) Pericyte-targeting prodrug overcomes tumor resistance to vascular disrupting agents. J Clin Invest 127(10):3689-3701

8. Ruan J, Luo M, Wang C et al (2013) Imatinib disrupts lymphoma angiogenesis by targeting vascular pericytes. Blood 121(26):5192-5202

9. Owens LV, Xu L, Craven RJ et al (1995) Overexpression of the focal adhesion kinase (p125FAK) in invasive human tumors. Cancer Res 55(13):2752-2755

10. Schmitz KJ, Grabellus F, Callies R et al (2005) High expression of focal adhesion kinase (p125FAK) in node-negative breast cancer is related to overexpression of HER-2/neu and activated Akt kinase but does not predict outcome. Breast Cancer Res 7(2):R194-203

11. Ji HF, Pang D, Fu SB et al (2013) Overexpression of focal adhesion kinase correlates with increased lymph node metastasis and poor prognosis in non-small-cell lung cancer. J Cancer Res Clin Oncol 139(3):429-435

12. Tavora B, Batista S, Reynolds LE et al (2010) Endothelial FAK is required for tumour angiogenesis. EMBO Mol Med 2(12):516-528

13. Sulzmaier FJ, Jean C, Schlaepfer DD (2014) FAK in cancer: mechanistic findings and clinical applications. Nat Rev Cancer 14(9):598-610

14. Lechertier T, Hodivala-Dilke K (2012) Focal adhesion kinase and tumour angiogenesis. J Pathol 226(2):404-412

15. Lee J, Borboa AK, Chun HB et al (2010) Conditional deletion of the focal adhesion kinase FAK alters remodeling of the bloodbrain barrier in glioma. Cancer Res 70(24):10131-10140

16. Corsi JM, Houbron C, Billuart P et al (2009) Autophosphorylation-independent and -dependent functions of focal adhesion kinase during development. J Biol Chem 284(50):34769-34776

17. Pedrosa AR, Bodrug N, Gomez-Escudero J et al (2019) Tumor angiogenesis is differentially regulated by phosphorylation of endothelial cell focal adhesion kinase tyrosines-397 and -861. Can Res 79(17):4371-4386

18. Tavora B, Reynolds LE, Batista S et al (2014) Endothelial-cell FAK targeting sensitizes tumours to DNA-damaging therapy. Nature 514(7520):112-116

19. Lechertier T, Reynolds LE, Kim H et al (2020) Pericyte FAK negatively regulates Gas6/Axl signalling to suppress tumour angiogenesis and tumour growth. Nat Commun 11(1):2810

20. Wong PP, Munoz-Felix JM, Hijazi M et al (2020) Cancer burden is controlled by Mural cell-beta3-integrin regulated crosstalk with tumor cells. Cell 181(6):1346-1363

21. Viski C, Konig C, Kijewska M et al (2016) Endosialin-expressing pericytes promote metastatic dissemination. Can Res 76(18):5313-5325

22. Murgai M, Ju W, Eason M et al (2017) KLF4-dependent perivascular cell plasticity mediates pre-metastatic niche formation and metastasis. Nat Med 23(10):1176-1190

23. Baluk P, Lee CG, Link $\mathrm{H}$ et al (2004) Regulated angiogenesis and vascular regression in mice overexpressing vascular endothelial growth factor in airways. Am J Pathol 165(4):1071-1085

24. Inai T, Mancuso M, Hashizume $H$ et al (2004) Inhibition of vascular endothelial growth factor (VEGF) signaling in cancer causes loss of endothelial fenestrations, regression of tumor vessels, and appearance of basement membrane ghosts. Am J Pathol 165(1):35-52 
25. Gilbert LA, Hemann MT (2010) DNA damage-mediated induction of a chemoresistant niche. Cell 143(3):355-366

26. Cao Z, Ding BS, Guo P et al (2014) Angiocrine factors deployed by tumor vascular niche induce $B$ cell lymphoma invasiveness and chemoresistance. Cancer Cell 25(3):350-365

27. Armulik A, Genove G, Mae M et al (2010) Pericytes regulate the blood-brain barrier. Nature 468(7323):557-561

28. Chen J, Luo Y, Hui H et al (2017) CD146 coordinates brain endothelial cell-pericyte communication for blood-brain barrier development. Proc Natl Acad Sci USA 114(36):E7622-E7631

29. Aplin AC, Fogel E, Nicosia RF (2010) MCP-1 promotes mural cell recruitment during angiogenesis in the aortic ring model. Angiogenesis 13(3):219-226

30. Gazzaniga S, Bravo AI, Guglielmotti A et al (2007) Targeting tumor-associated macrophages and inhibition of MCP-1 reduce angiogenesis and tumor growth in a human melanoma xenograft. J Invest Dermatol 127(8):2031-2041

31. Cranford TL, Velazquez KT, Enos RT et al (2017) Loss of monocyte chemoattractant protein-1 expression delays mammary tumorigenesis and reduces localized inflammation in the C3(1)/ SV40Tag triple negative breast cancer model. Cancer Biol Ther 18(2):85-93

32. Roberts WG, Ung E, Whalen P et al (2008) Antitumor activity and pharmacology of a selective focal adhesion kinase inhibitor, PF-562,271. Can Res 68(6):1935-1944

33. Duxbury MS, Ito H, Zinner MJ et al (2004) Focal adhesion kinase gene silencing promotes anoikis and suppresses metastasis of human pancreatic adenocarcinoma cells. Surgery 135(5):555-562

34. Stokes JB, Adair SJ, Slack-Davis JK et al (2011) Inhibition of focal adhesion kinase by PF-562,271 inhibits the growth and metastasis of pancreatic cancer concomitant with altering the tumor microenvironment. Mol Cancer Ther 10(11):2135-2145

35. Alexopoulou AN, Lees DM, Bodrug N et al (2017) Focal Adhesion Kinase (FAK) tyrosine 397E mutation restores the vascular leakage defect in endothelium-specific FAK-kinase dead mice. J Pathol 242(3):358-370

36. Huang FJ, You WK, Bonaldo P et al (2010) Pericyte deficiencies lead to aberrant tumor vascularizaton in the brain of the NG2 null mouse. Dev Biol 344(2):1035-1046

37. Cooke VG, LeBleu VS, Keskin D et al (2012) Pericyte depletion results in hypoxia-associated epithelial-to-mesenchymal transition and metastasis mediated by met signaling pathway. Cancer Cell 21(1):66-81

38. Yoshida S, Kawai H, Eguchi T et al (2019) Tumor angiogenic inhibition triggered necrosis (TAITN) in oral cancer. Cells 8:7

39. Ramanujan S, Koenig GC, Padera TP et al (2000) Local imbalance of proangiogenic and antiangiogenic factors: a potential mechanism of focal necrosis and dormancy in tumors. Can Res 60(5):1442-1448

40. Acharyya S, Oskarsson T, Vanharanta S et al (2012) A CXCL1 paracrine network links cancer chemoresistance and metastasis. Cell 150(1):165-178

41. Armstrong LC, Bjorkblom B, Hankenson KD et al (2002) Thrombospondin 2 inhibits microvascular endothelial cell proliferation by a caspase-independent mechanism. Mol Biol Cell 13(6): 1893-1905

42. Li M, Knight DA, As L et al (2013) A role for CCL2 in both tumor progression and immunosurveillance. Oncoimmunology. 2(7):25474

43. Yang X, Qiao D, Meyer K et al (2012) Angiogenesis induced by signal transducer and activator of transcription 5A (STAT5A) is dependent on autocrine activity of proliferin. $\mathrm{J}$ Biol Chem 287(9):6490-6502

44. Carpen T, Sorsa T, Jouhi L et al (2019) High levels of tissue inhibitor of metalloproteinase-1 (TIMP-1) in the serum are associated with poor prognosis in HPV-negative squamous cell oropharyngeal cancer. Cancer Immunol Immunother 68(8):1263-1272

45. Takahara M, Nagato T, Komabayashi Y et al (2013) Soluble ICAM-1 secretion and its functional role as an autocrine growth factor in nasal NK/T cell lymphoma cells. Exp Hematol 41(8):711-718

46. Tang Z, Gillatt D, Rowe E et al (2019) IGFBP-2 acts as a tumour suppressor and plays a role in determining chemosensitivity in bladder cancer cells. Oncotarget 10(66):7043-7057

47. Folkman J (2006) Antiangiogenesis in cancer therapy-endostatin and its mechanisms of action. Exp Cell Res 312(5):594-607

48. Vazquez F, Hastings G, Ortega MA et al (1999) METH-1, a human ortholog of ADAMTS-1, and METH-2 are members of a new family of proteins with angio-inhibitory activity. J Biol Chem 274(33):23349-23357

49. Sandilands E, Serrels B, McEwan DG et al (2011) Autophagic targeting of Src promotes cancer cell survival following reduced FAK signalling. Nat Cell Biol 14(1):51-60

50. Beausejour M, Noel D, Thibodeau S et al (2012) Integrin/Fak/ Src-mediated regulation of cell survival and anoikis in human intestinal epithelial crypt cells: selective engagement and roles of PI3-K isoform complexes. Apoptosis 17(6):566-578

51. Lim ST, Chen XL, Tomar A et al (2010) Knock-in mutation reveals an essential role for focal adhesion kinase activity in blood vessel morphogenesis and cell motility-polarity but not cell proliferation. J Biol Chem 285(28):21526-21536

52. Tavora B, Batista S, Alexopoulou AN et al (2014) Generation of point-mutant FAK knockin mice. Genesis 52(11):907-915

53. Tigges U, Welser-Alves JV, Boroujerdi A et al (2012) A novel and simple method for culturing pericytes from mouse brain. Microvasc Res 84(1):74-80

54. Reynolds LE, D'Amico G, Lechertier T et al (2017) Dual role of pericyte alpha6beta1-integrin in tumour blood vessels. J Cell Sci 130(9):1583-1595

55. Baker M, Robinson SD, Lechertier T et al (2012) Use of the mouse aortic ring assay to study angiogenesis. Nat Protoc 7(1):89-104

Publisher's Note Springer Nature remains neutral with regard to jurisdictional claims in published maps and institutional affiliations. 